\title{
One-Fold Anisotropy of Silver Chiral Nanoparticles Studied by Second Harmonic Generation
}

Bin Dong, ${ }^{\text {a,b } \ddagger}$ Junjun Liu, ${ }^{\mathrm{c}, \mathrm{d} \ddagger}$ Man Xue, ${ }^{\mathrm{a}, \mathrm{b}}$ Ziyue Ni, ${ }^{\mathrm{c}}$ Yuan Guo, ${ }^{\mathrm{a}, \mathrm{b}}$ Zhifeng Huang, ${ }^{\text {c,d,e }}$ and Zhen Zhang ${ }^{* a, b}$

${ }^{a}$ Beijing National Laboratory of Molecular Sciences, State Key Laboratory of Molecular Reaction Dynamics, CAS Research/Education Center for Excellence in Molecular Sciences, Institute of Chemistry, Chinese Academy of Sciences, Beijing 100190, China

${ }^{\mathrm{b}}$ University of the Chinese Academy of Sciences, Beijing 100049, China

'Department of Physics, Hong Kong Baptist University (HKBU), Kowloon Tong, Kowloon, Hong Kong SAR, China

${ }^{\mathrm{d}}$ HKBU Institute of Research and Continuing Education, Shenzhen, Guangdong 518057, China

${ }^{\mathrm{e}}$ Institute of Advanced Materials, State Key Laboratory of Environmental and Biological Analysis, Golden Meditech Centre for NeuroRegeneration Sciences, HKBU, Kowloon Tong, Kowloon, Hong Kong SAR, China

*B.D. and J.J.L contributed equally.

*corresponding author: Dr. Zhifeng Huang, Email: zfhuang@hkbu.edu.hk; Prof. Zhen Zhang, Email: zhangz@iccas.ac.cn 


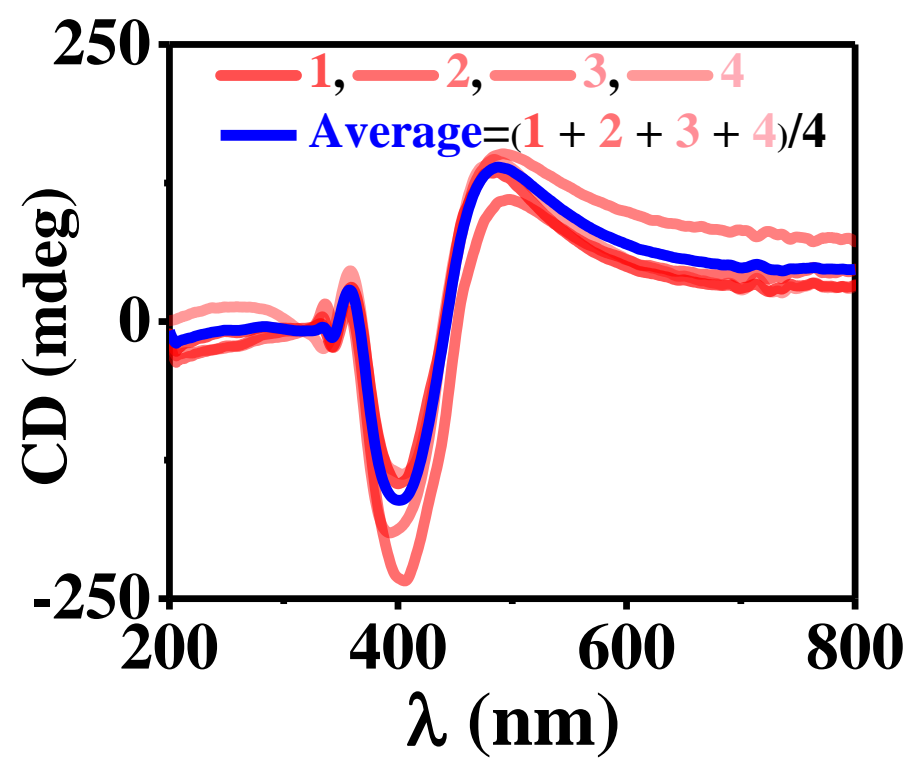

Figure S1. CD Characterization of the LH-Ag CNPs with $P=50 \mathrm{~nm}$ and $n=1$. Four CD spectra (spectrum 1 - 4) were subsequently recorded, and after monitoring each CD spectrum the sample was manually rotated in an angle of $90^{\circ}$ around its normal axis before measuring the next $\mathrm{CD}$ spectrum. Then the four $\mathrm{CD}$ spectra were algebraically averaged to obtain a CD spectrum of the sample (blue line), to eliminate linear birefringence induced by the growing orientation of Ag CNPs on the supporting substrate.
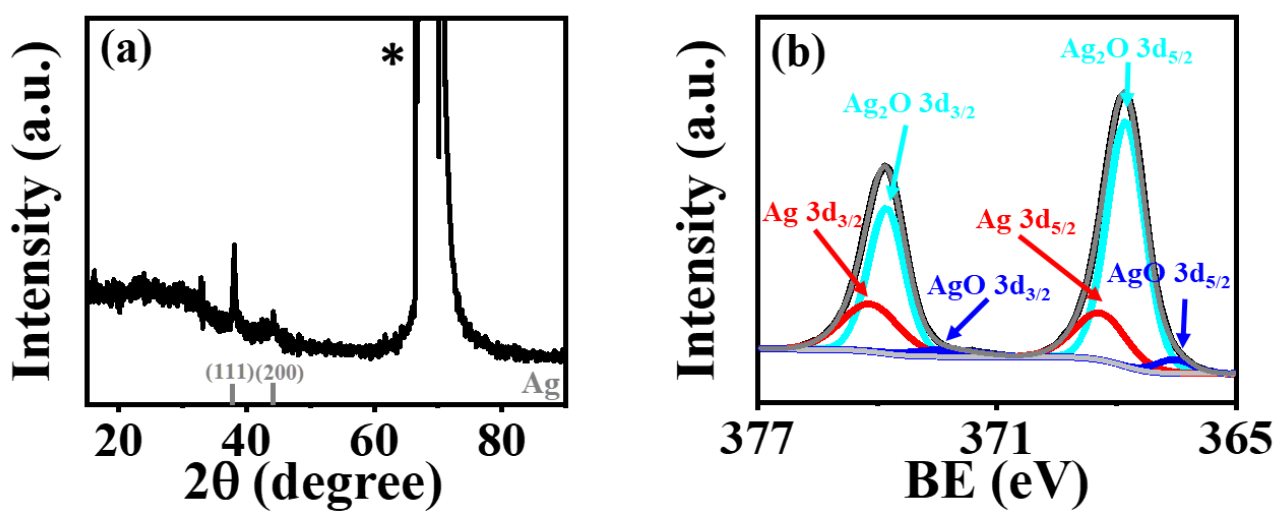

Figure S2. Characterization of RH-Ag CNPs ( $P=50 \mathrm{~nm}$ and $n=1$ ) with (a) XRD and (b) XPS (Ag 3d). (a) The peak marked by an asterisk is assigned to Si wafer on which the RH-Ag CNPs were deposited. 


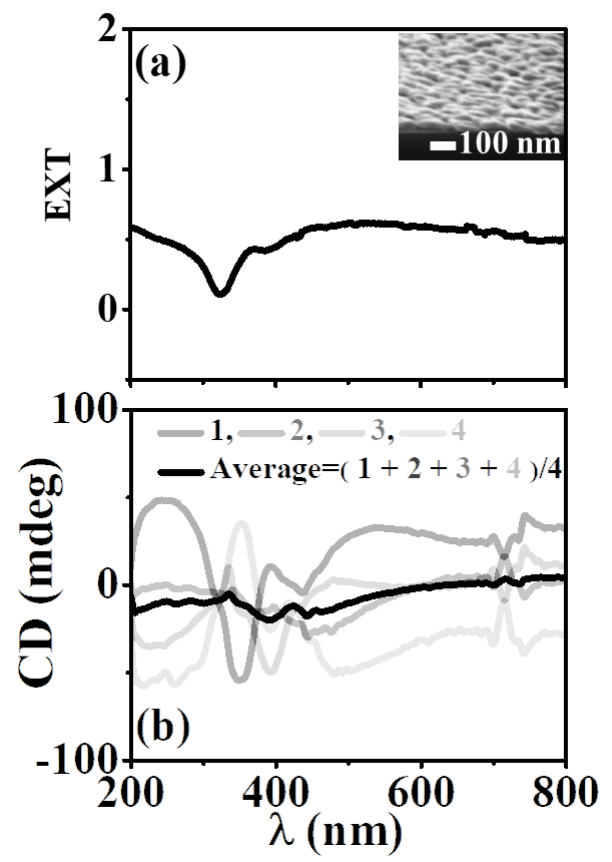

Figure S3. UV/visible spectra of the close-packed array of achiral Ag NPs with a nominal thickness of 40 nm: (a) extinction; (b) Four CD spectra (spectrum 1-4) were subsequently recorded, and after monitoring each CD spectrum, the sample was manually rotated in an angle of $90^{\circ}$ around its normal axis before measuring the next $\mathrm{CD}$ spectrum. Then the four $\mathrm{CD}$ spectra were algebraically averaged to obtain a CD spectrum of the sample. No overlapping of spectra 1-4 and non-zero CD signals in the mean CD spectrum can be attributed to linear birefringence resulting from the tilted protrusion of achiral Ag NPs, which is characterized in the inset SEM image.
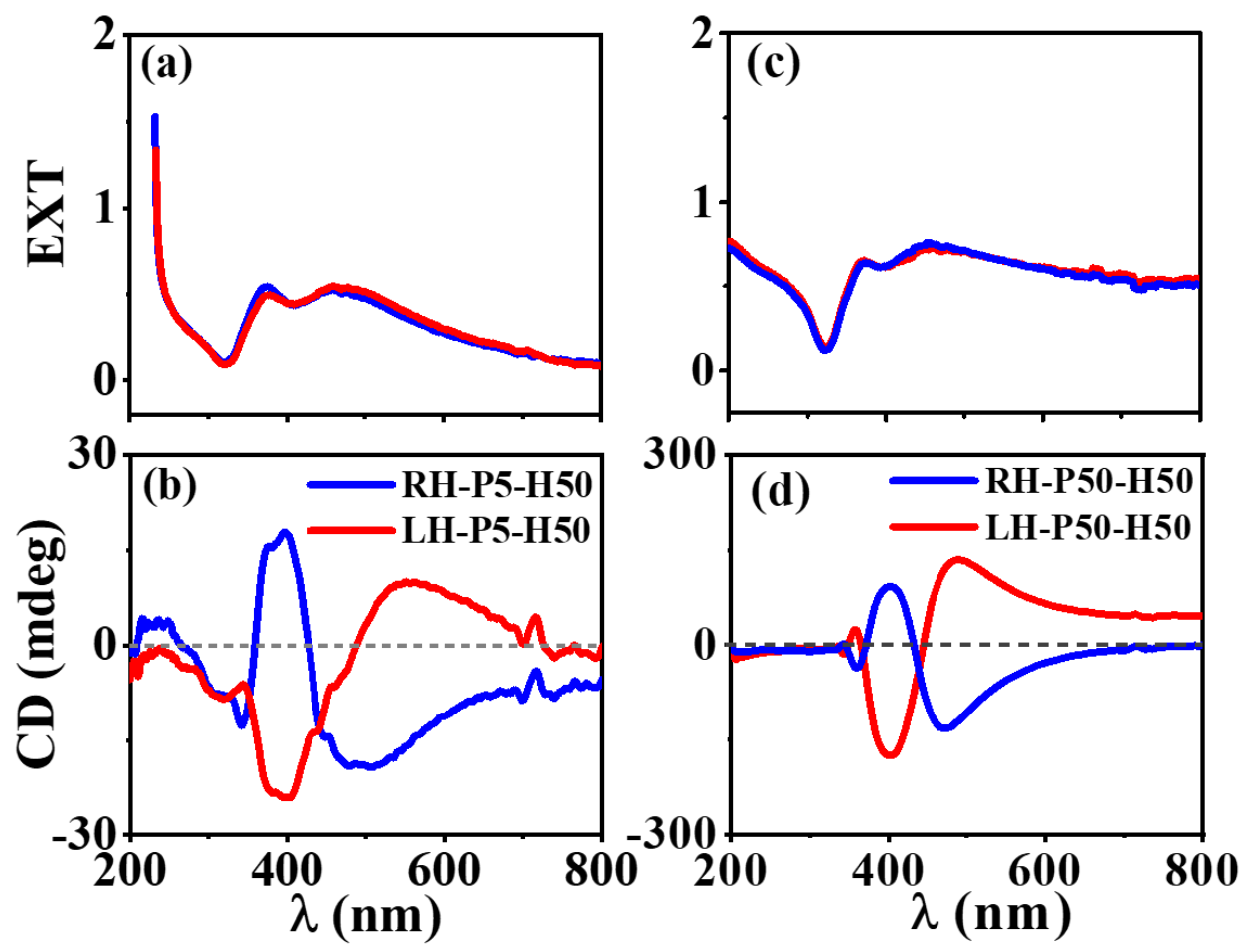

Figure S4. GLAD of Ag CNPs with (a, b) $P=5 \mathrm{~nm}$ and $n=10$, (c, d) $P=50 \mathrm{~nm}$ and $n=1$. UV/visible spectra: (a, c) extinction; (b, d) CD. Blue lines: RH-Ag CNPs; red lines: LH-Ag CNPs. 


\section{The SHG intensity theory of the Ag CNPs with different pitches}

We have noted that the SHG intensity of Ag CNPs with $P=15 \mathrm{~nm}$ is stronger than ones with $P=25$ nm or $P=50 \mathrm{~nm}$ as shown in figure 3 , while the linear extinction spectra show similar intensity or in case of $P=50 \mathrm{~nm}$ larger extinction cross sections. We have known that enhanced SHG occurs in the small metal nanoparticles that illuminated with light near resonant with the localized surface plasmon frequency of the metal structure. ${ }^{1}$ The localized surface plasmon resonance (LSPR) depends on the size and shape of the particles. ${ }^{2-3}$ Richard Gans predicted the splitting of surface plasmons into two distinct modes due to surface curvature and symmetry in $2006 .{ }^{4}$ The extinction cross-section for NPs of various shapes can be calculated as:

$$
C_{e x t}=\frac{2 \pi V}{3 \lambda} \varepsilon_{m}^{3 / 2} \sum_{j} \frac{\left(1 / P_{j}^{2}\right) \varepsilon_{i}}{\left(\varepsilon_{r}+\left(1-P_{j} / P_{j}\right) \varepsilon_{m}\right)^{2}+\varepsilon_{i}^{2}}
$$

Where $V$ is the volume of the particle and $P_{j}$ is the depolarization factor which can be described as:

$$
\begin{aligned}
P_{\text {height }} & =\frac{1-e^{2}}{e^{2}}\left[\frac{1}{2 e} \ln \left(\frac{1+e}{1-e}\right)-1\right] \\
P_{\text {width }} & =\frac{1-P_{\text {height }}}{2}
\end{aligned}
$$

$e$ is ellipticity given by:

$$
e^{2}=1-\left(\frac{\text { height }}{\text { width }}\right)^{-2}
$$

As it showed in table S1, we have counted the averaged width and height of the nanoparticles from the SEM images in Figure 1 in the manuscript. Then we calculated the cross-section values of AgNPs with the dependence of height and width as it showed below.

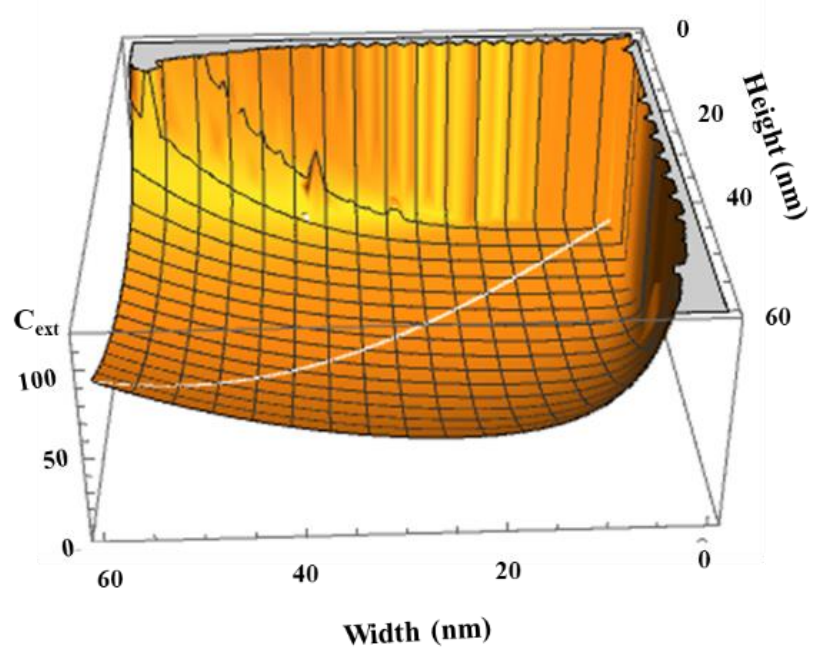

Figure S5. The calculated value of $C_{e x t}$ and the size of nanoparticle.

The size and the shape of particles were determined by SEM images in figure 3. 
Table S1. The average widths with different height of NPs.

\begin{tabular}{|c|c|c|c|c|}
\hline Pitch $(\mathrm{nm})$ & 5 & 15 & 25 & 50 \\
\hline Width $(\mathrm{nm})$ & $25.5 \pm 5$ & $34.1 \pm 11$ & $49.9 \pm 10$ & $60.4 \pm 16$ \\
\hline Height $(\mathrm{nm})$ & $9.1 \pm 2$ & $18.8 \pm 7$ & $26.8 \pm 4$ & $46.6 \pm 5$ \\
\hline
\end{tabular}

Therefore, we plotted the $C_{e x t}$ as the function of the width and height in Figure S6.

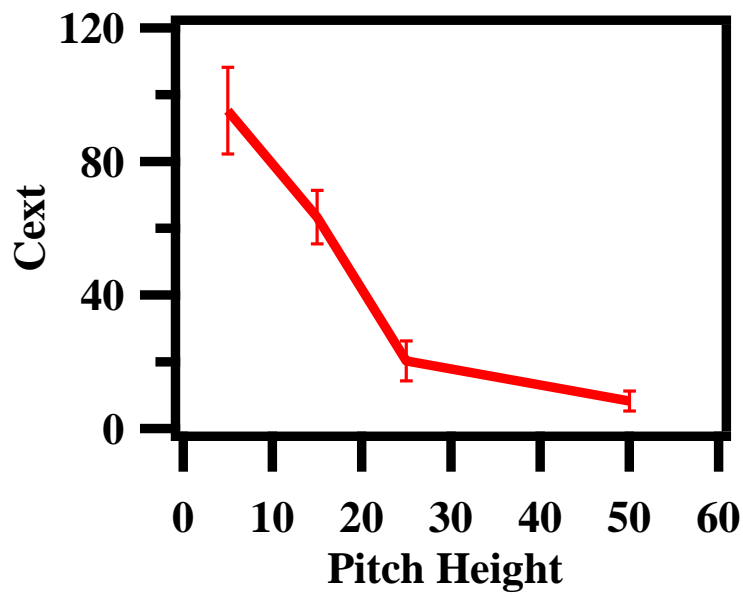

Figure S6. Value of $C_{e x t}$ at the average width depends on the pitch heights.

The value of $\mathrm{C}_{\mathrm{ext}}$ at $P=15 \mathrm{~nm}$ is about 2 times higher than one of $P=25 \mathrm{~nm}$ and $50 \mathrm{~nm}$ indicated that the $P=15 \mathrm{~nm}$ nanoparticles could generate stronger SH signals. But for the $5 \mathrm{~nm}$ pitch nanoparticle, we found that the $C_{\text {ext }}$ we calculated gave the abnormal large signal which is not consistent with our experiment. So that the eq(4) are not suitable here. It has been known that the particles size is below $10 \mathrm{~nm}$, a so-called quantum plasmon resonances has to be considered. ${ }^{5}$ We will not go into the detailed discussion for this issue, but we have known that a strong blue shift of the LSPR with decreasing size for the small pitch nanoparticles. Therefore, we can assume that the LSPR of the $5 \mathrm{~nm}$ pitch height nanoparticles will shifted to the blue side that could make the SH signal weaker that the sizes that are larger than $10 \mathrm{~nm}$.

\section{References}

1. Moskovits, M., SURFACE-ENHANCED SPECTROSCOPY. Rev. Mod. Phys. 1985, 57 (3), 783-826.

2. Deka, G.; Sun, C.-K.; Fujita, K.; Chu, S.-W., Nonlinear plasmonic imaging techniques and their biological applications. Nanophotonics 2017, 6 (1), 31-49.

3. Singh, A. K.; Senapati, D.; Neely, A.; Kolawole, G.; Hawker, C.; Ray, P. C., Nonlinear optical properties of triangular silver nanomaterials. Chem. Phys. Lett. 2009, 481 (1-3), 94-98.

4. Petryayeva, E.; Krull, U. J., Localized surface plasmon resonance: Nanostructures, bioassays and biosensing-A review. Anal. Chim. Acta 2011, 706 (1), 8-24. 
5. Campos, A.; Troc, N.; Cottancin, E.; Pellarin, M.; Weissker, H.-C.; Lermé, J.; Kociak, M.; Hillenkamp, M., Plasmonic quantum size effects in silver nanoparticles are dominated by interfaces and local environments. Nat. Phys. 2019, 15 (3), 275-280. 